\title{
An optimal servo system based on sliding mode for the contact force of an active pantograph
}

\author{
Myat Thiri Ko*, Makoto YOKOYAMA** and Shun NAGAYOSHI* \\ *Graduate School of Science and Technology, Niigata University \\ 8050 Ikarashi 2nocho, Nishi-ku, Niigata 950-2181, Japan \\ **Niigata University \\ 8050 Ikarashi 2nocho, Nishi-ku, Niigata 950-2181, Japan \\ E-mail: m.yoko@eng.niigata-u.ac.jp
}

Received: 4 March 2017; Revised: 12 June 2017; Accepted: 28 July 2017

\begin{abstract}
This paper presents a design method of optimal servo systems based on sliding mode for an active pantograph with flexibility subject to the stiffness variation of the catenary. It is pointed out through our analysis that the flexibility makes the control problem much harder, because it increases not only the freedom of motion but also the relative degree between the control input and the contact force to be controlled. However, it is shown by employing the optimal control theory, especially SRL (Symmetric Root Locus), together with sliding mode theory that pole-zero cancellations play an important role in the controller design. Some numerical simulations in a practical situation, where the actuator dynamics and sensor noise are taken into account, show the effectiveness of the proposed design method.
\end{abstract}

Key words : Active pantograph, Sliding mode controller, Servo system, Sliding mode observer, Optimal control

\section{Introduction}

For high-speed trains, active control of the pantograph is crucial technology to collect electrical current from the overhead contact wire supported by vertical droppers, hangers and cantilevers (catenary system, as shown in Fig. 1). The contact force variation can cause contact losses, electric arc formations and sparking. This deteriorates the quality of current collection and increases the electrical related wear, and is one of the limiting factors for the maximum train speed. Therefore, maintaining the contact force in an admissible region is crucial for high speed trains and thus modelling and control of active pantograph-catenary systems have been taken much attention from many researchers. So far, some models and controllers for the system have been proposed. For example, Arnold and Simeon developed a rather rigorous model with PDEs and DAEs and then proposed a numerical solution method (Arnold and Simeon, 2000), Makino et al. developed a wing-speed low-noise collector and proposed an $H_{\infty}$ controller with disturbance observer (Makino et al., 1997), Yamashita et al. developed a low-noise active pantograph, and then applied a PID controller or an impedance control method (Yamashita et al., 2011), Chatter et al. proposed a controller based on the back-stepping method together with a high-gain observer (Chater et al., 2013), Allota, Pisano, et al. proposed higher order sliding mode controllers (Allotta et al., 2005), (Pisano and Usai, 2008) and (Pisano and Usai, 2004), Sanchez-Rebollo et al. proposed a hardware-in-the-loop strategy with a PID controller (Sanchez-Rebolloa et al., 2013).

In order to regulate the contact force, the authors and the Railway Technical Research Institute have developed an active pantograph using a pneumatic actuator, and presented modelling and a robust regulator with a rigid frame model (Yokoyama et al., 2015). The points for controller design in that paper can be summarized as follows:

"If the contact force can be used as a switching function in the sliding mode controller, the contact force would be regulated very well because sliding motion takes place when the switching function is forced to be zero by a relay control. In such a case, however, it is impossible to guarantee the existence of sliding mode because of the relative degree between the control input and the contact force, which must be one for the existence. In order to get around this problem, the switching function was constructed just to "approximate” the contact force by introducing a design parameter." 
After that, however, it was found through some experiments that the frame had flexibility which could not be ignored to control the contact force. This flexibility increases not only the freedom of motion but also the relative degree mentioned above.

In this paper, therefore, we present a design method of optimal servo systems based on sliding mode taking account of the flexibility. Although most of the active pantograph systems in literature only regulate the contact force under model uncertainty or disturbance, servo systems should be realized by making efficient use of active force. For example, the desired contact force might be changed due to some trouble in the catenary. In such a situation, transient response to a step command would be very important.

LQR (Linear Quadratic Regulator) technique has been successively applied to not only linear servo systems but also sliding mode control systems. To our best knowledge, however, the reference signal must be assumed to be zero to treat it as a regulator design problem in the optimal design methods for sliding mode servo controllers (Shtessel et al. 2014, Myat Thiri Ko et al., 2016b). As a result, transient response such as step response cannot be taken into account explicitly. On the other hand, Takeda and Kitamori proposed a design method of linear optimal servo systems without such an assumption (Takeda and Kitamori, 1977). We apply their method to design a sliding mode servo controller, which makes it possible to take the transient response into account and gives an important guideline for controller design. Finally, we combine the controller with a sliding mode observer (Myat Thiri Ko et al., 2016a,b)

This paper is organized as follows: a mathematical model and analysis of the active pantograph are described in section 2, section 3 is devoted to the design of the controller/observer and analysis of the optimal closed-loop system, while simulation results are given in section 4 , and the last section concludes this work.

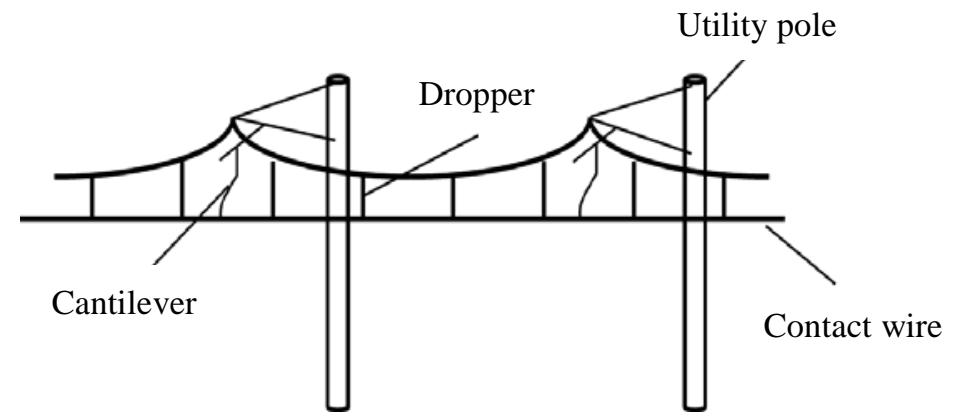

Fig. 1 Schematic of a typical catenary system. The catenary mainly consists of the contact wire, and some supporters such as droppers and cantilevers to keep the contact wire in a certain shape at certain positions, which give rise to perturbation with respect to the equivalent stiffness of the catenary.

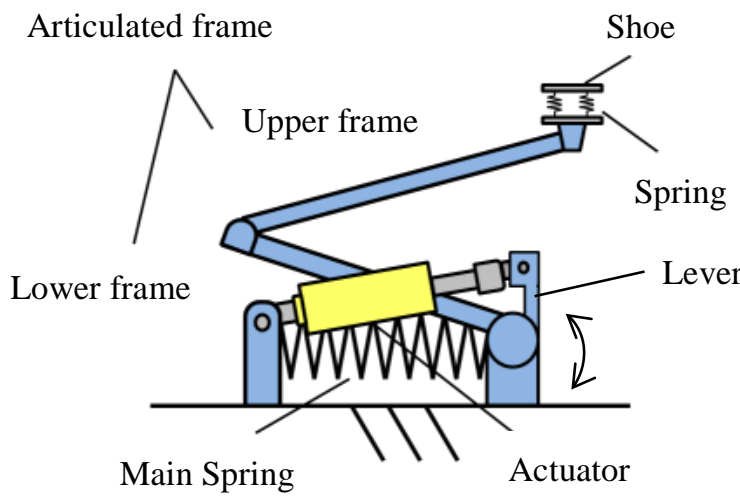

Fig. 2 Schematic of the experimental active pantograph developed by Railway Technical Research Institute. The force generated by the actuator or the main spring is transformed to torque around the heel of the lower frame by the lever to rotate the articulated frame, and thus the entire pantograph can be lifted. The main spring is originally for a passive pantograph and not used for active control, however, may be used for fail-safe. 


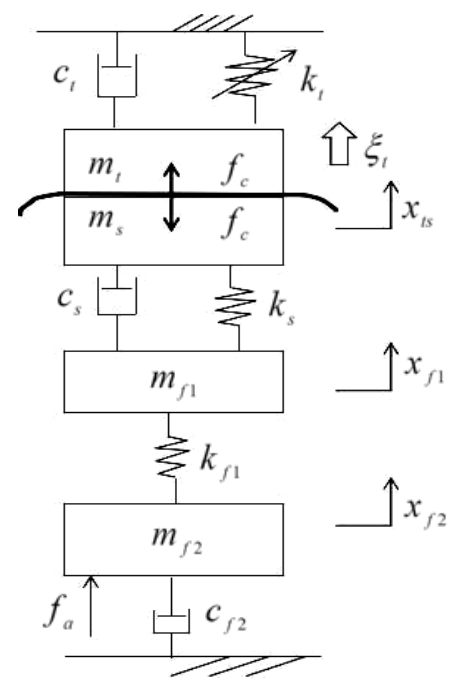

Fig. 3 Pantograph model with flexibility of the lower frame which was found in our experiment for identification.

\begin{tabular}{|c|c|c|c|c|c|}
\hline \multicolumn{6}{|c|}{ Table 1 Nomenclature } \\
\hline catenary stiffness & $k_{t}$ & $1100 \mathrm{~N} / \mathrm{m}$ & catenary damping & $c_{t}$ & $100 \mathrm{Ns} / \mathrm{m}$ \\
\hline mass of catenary & $m_{t}$ & $100 \mathrm{~kg}$ & & & \\
\hline shoe-upper frame stiffness & $k_{s}$ & $38000 \mathrm{~N} / \mathrm{m}$ & $\begin{array}{c}\text { shoe-upper frame } \\
\text { damping }\end{array}$ & $c_{s}$ & $60 \mathrm{Ns} / \mathrm{m}$ \\
\hline mass of shoe & $m_{s}$ & $2.13 \mathrm{~kg}$ & mass of upper frame & $m_{f 1}$ & $6 \mathrm{~kg}$ \\
\hline upper frame-lower frame stiffness & $k_{f 1}$ & $19218 \mathrm{~N} / \mathrm{m}$ & $\begin{array}{l}\text { upper frame-lower } \\
\text { frame damping }\end{array}$ & $c_{f 1}$ & $\simeq 0 \mathrm{Ns} / \mathrm{m}$ \\
\hline mass of lower frame & $m_{f 2}$ & $10 \mathrm{~kg}$ & lower frame damping & $c_{f 2}$ & $80 \mathrm{Ns} / \mathrm{m}$ \\
\hline contact force & $f_{c}$ & {$[\mathrm{~N}]$} & control force & $f_{a}$ & {$[\mathrm{~N}]$} \\
\hline $\begin{array}{l}\text { displacement of } \\
\text { contact wire/shoe }\end{array}$ & $x_{t s}$ & {$[\mathrm{~m}]$} & $\begin{array}{c}\text { displacement of } \\
\text { upper frame }\end{array}$ & $x_{f 1}$ & {$[\mathrm{~m}]$} \\
\hline $\begin{array}{l}\text { displacement of } \\
\text { lower frame }\end{array}$ & $x_{f 2}$ & {$[\mathrm{~m}]$} & $\begin{array}{c}\text { uncertainty/disturbance } \\
\text { due to catenary's } \\
\text { stiffness variation }\end{array}$ & $\xi_{t}$ & {$[\mathrm{~N}]$} \\
\hline
\end{tabular}

\section{Mathematical model and analysis of the plant}

A schematic of the active pantograph we have developed is shown in Fig. 2. Taking account of the flexibility in the frame, a three degree of freedom model is used as shown in Fig. 3, where $m_{t}, m_{s}, m_{f 1}$ and $m_{f 2}$ are masses of the overhead wire, the pantograph head, the upper frame and the lower frame, respectively, $f_{a}$ is control force generated by the pneumatic actuator (the other variables are defined as shown in table 1). Assuming that the contact wire and the shoe on the pantograph head are connected all the time, and that the origin in the coordinates is the equilibrium point, the equations of motion of the masses are given by 


$$
\begin{aligned}
& \left(m_{t}+m_{s}\right) \ddot{x}_{t s}=-\left(c_{t}+c_{s}\right) \dot{x}_{t s}-\left(k_{t}+k_{s}\right) x_{t s}+c_{s} \dot{x}_{f 1}+k_{s} x_{f 1}+\xi_{t} \\
& m_{f 1} \ddot{x}_{f 1}=c_{s} \dot{x}_{t s}+k_{s} x_{t s}-\left(c_{s}+c_{f 1}\right) \dot{x}_{f 1}-\left(k_{s}+k_{f 1}\right) x_{f 1}+c_{f 1} \dot{x}_{f 2}+k_{f 1} x_{f 2} \\
& m_{f 2} \ddot{x}_{f 2}=c_{f 1} \dot{x}_{f 1}+k_{f 1} x_{f 1}-\left(c_{f 1}+c_{f 2}\right) \dot{x}_{f 2}-k_{f 1} x_{f 2}+f_{a}
\end{aligned}
$$

where $\xi_{t} \triangleq_{\Delta} k_{t} x_{t s}$ is the uncertainty/disturbance due to the change of the equivalent stiffness $k_{t}$ of the catenary system. Taking $\mathbf{x}=\left[\begin{array}{llllll}x_{t s} & \dot{x}_{t s} & x_{f 1} & \dot{x}_{f 1} & x_{f 2} & \dot{x}_{f 2}\end{array}\right]^{T}$ as the state vector, it follows from Eqs. (1)-(3), the state equation

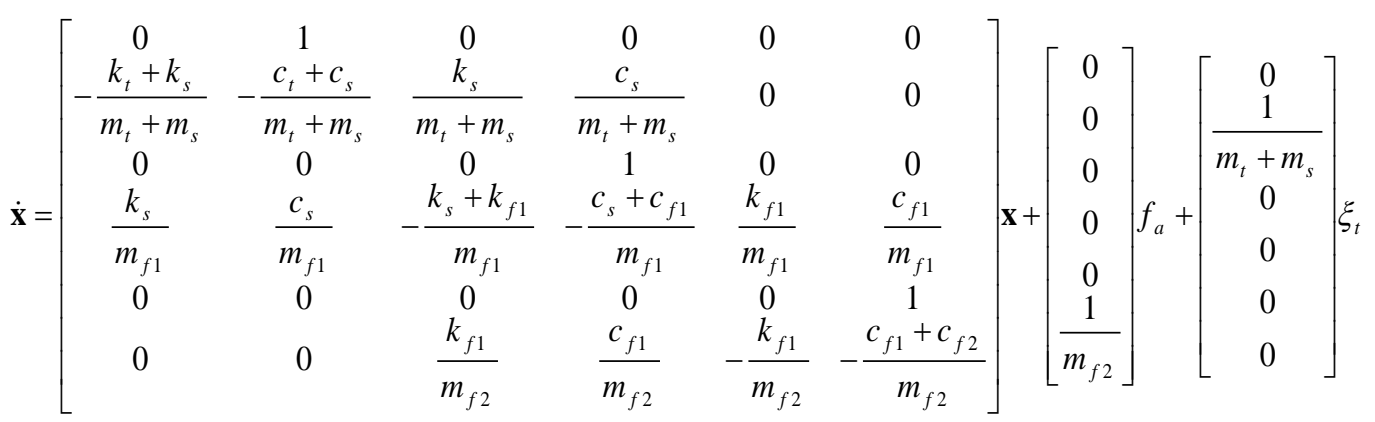

Since the contact force includes inertial force of the contact wire and pantograph head, in order to obtain an expression of the contact force we need the following equations of motion with respect to each mass independently.

$$
\begin{aligned}
& m_{t} \ddot{x}_{t}=-c_{t} \dot{x}_{t}-k_{t} x_{t}+f_{c}-\Delta k_{t} x_{t} \\
& m_{s} \ddot{x}_{s}=-c_{s}\left(\dot{x}_{s}-\dot{x}_{f 1}\right)-k_{s}\left(x_{s}-x_{f 1}\right)-f_{c}
\end{aligned}
$$

From these equations, it follows that the contact force can be represented by

$$
\begin{aligned}
f_{c} & =\frac{1}{m_{t}+m_{s}}\left[k_{t} m_{s}-m_{t} k_{s} \quad c_{t} m_{s}-m_{t} c_{s} \quad m_{t} k_{s} \quad m_{t} c_{s} \quad 0 \quad 0\right] \mathbf{x}-\frac{m_{s}}{m_{t}+m_{s}} \xi_{t} \\
& =\mathbf{c x}+d \xi_{t}
\end{aligned}
$$

It should be noted that the relative degree between the uncertainty $\xi_{t}$ and the contact force $f_{c}$ is zero because the uncertainty $\xi_{t}$ appears in the output equation (7).

With the state equation (4) and output equation (7), the zeros of the transfer function from the control force to the contact force are values of $s$ satisfying the determinant equation

$$
\left|\begin{array}{cc}
\mathbf{A}-s \mathbf{I} & \mathbf{b} \\
\mathbf{c} & 0
\end{array}\right|=\left(m_{t} s^{2}+c_{t} s+k_{t}\right)\left(c_{s} s+k_{s}\right)\left(c_{f 1} s+k_{f 1}\right)=0
$$

where $c_{f 1} \simeq 0$ from our identification experiments, and thus the zeros are obtained as follows:

$$
s=-\frac{k_{s}}{c_{s}}, \frac{-c_{t} \pm \sqrt{c_{t}^{2}-4 m_{t} k_{t}}}{2 m_{t}}
$$

which reveals an important fact that two complex zeros of the transfer function are the same as the poles of the nominal catenary subsystem given by (5), and that the relative degree is three. If $c_{f 1} \neq 0$, the relative degree would be four. On the other hand, the relative degree of the transfer function from the uncertainty $\xi_{t}$ to the contact force is zero as mentioned above. In general output regulation or disturbance rejection problems, the relative degree and pole-zero cancellation play an important role in controller design. That is, from the above observations, we can see that it must be very difficult to reject the influence of the uncertainty completely, because the relative degree of the transfer function 
from the control input to the contact force is less than that of the transfer function from the uncertainty. Nevertheless, in order to reduce the influence of the uncertainty on the contact force, some of the closed-loop poles should be assigned in exactly the same location as the catenary poles, yielding pole-zero cancellation. This will be discussed in the next section with some discussions about analysis of the closed-loop system again.

\section{Controller design}

We employ sliding mode theory to design both controller and observer. The overall block diagram is shown in Fig. 4, where $r$ is the reference contact force, $\sigma$ is the switching function, and $u$ is the control input. To compensate the tracking error in steady state, integral action with respect to the tracking error is used, although the tracking error cannot be kept zero due to the uncertainty as mentioned above. At first, assuming that all the state variables are available for control, we design an optimal sliding mode control law applying a design method of optimal linear tracking systems (Takeda and Kitamori, 1977), and then design a sliding mode observer to estimate the state.

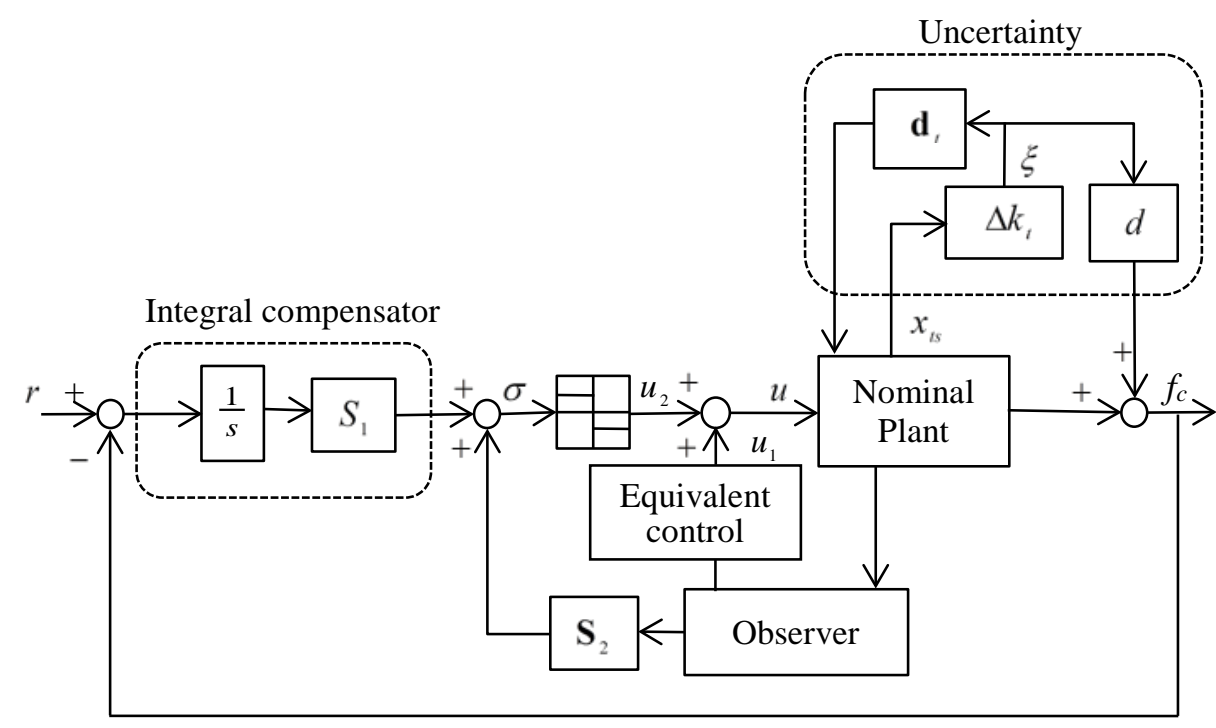

Fig. 4 Overall block diagram of the proposed optimal servo system including an integral compensator to achieve zero steady state error to a step command without the uncertainty. The observer does not use the contact force $f_{c}$ which is the controlled variable, because it is directly affected by the uncertainty and would deteriorate the estimation.

\subsection{Optimal sliding mode control law}

Having introduced the integral action, we define the augmented state vector, and rewrite the state equation, Eq. (4) and the output equation (7) as

$$
\begin{aligned}
& \mathbf{z}=\left[\begin{array}{lllllll}
\int r-f_{c} & x_{t s} & \dot{x}_{t s} & x_{f 1} & \dot{x}_{f 1} & x_{f 2} & \dot{x}_{f 2}
\end{array}\right] \\
& \dot{\mathbf{z}}=\left[\begin{array}{cc}
0 & -\mathbf{c} \\
0 & \mathbf{A}
\end{array}\right] \mathbf{z}+\left[\begin{array}{l}
0 \\
\mathbf{b}
\end{array}\right] u+\left[\begin{array}{l}
1 \\
\mathbf{0}
\end{array}\right] r+\left[\begin{array}{l}
d \\
\mathbf{d}_{t}
\end{array}\right] \xi_{t} \\
& =\overline{\mathbf{A}} z+\overline{\mathbf{b}} u+\overline{\mathbf{v}} r+\overline{\mathbf{d}} \xi_{t} \\
& f_{c}=\left[\begin{array}{ll}
0 & \mathbf{c}
\end{array}\right] \mathbf{z}+d \xi_{t}=\overline{\mathbf{c}} \mathbf{z}+d \xi_{t}
\end{aligned}
$$

Based on the standard sliding mode control theory, the control input $u$ is composed of two components as follows:

$$
\begin{aligned}
& u=u_{1}+u_{2} \\
& u_{1}=-(\mathbf{S b})^{-1} \mathbf{S}[\overline{\mathbf{A}} \mathbf{z}-\overline{\mathbf{v}} r], u_{2}=(\mathbf{S} \overline{\mathbf{b}})^{-1} \rho \operatorname{sgn}(\sigma), \sigma=\mathbf{S z}=\left[\begin{array}{ll}
S_{1} & \mathbf{S}_{2}
\end{array}\right] \mathbf{z}
\end{aligned}
$$


where $\sigma$ is a switching function, $\rho$ is a relay gain, $u_{1}$ is the so called equivalent control which is obtained by solving $\dot{\sigma}=\mathbf{S} \dot{\mathbf{z}}=0$ with Eq. (10) for the control $u$, and $u_{2}$ is the relay input to bring about the sliding motion. It is very important how to determine the switching function $\sigma$ for a sliding mode controller, because the sliding motion is prescribed by the switching function; an ideal sliding motion can be obtained from $\sigma=\dot{\sigma}=0$. To simplify the switching function design, the so called regular form (Edwards et al., 1998) is employed because this special form makes it possible to avoid the use of complicated equivalent control to represent the dynamics during ideal sliding mode. To apply the regular form technique, the state equation (10) is divided into two subsystems, i.e. two sub-states $\mathbf{z}_{N}$ and $z_{R}$, which are sometimes referred to as "Null space dynamics" and "Range space dynamics" respectively.

$$
\begin{aligned}
& \dot{\mathbf{z}}=\left[\begin{array}{c}
\dot{\mathbf{z}}_{N} \\
\dot{z}_{R}
\end{array}\right]=\left[\begin{array}{cc}
\overline{\mathbf{A}}_{N 1} & \overline{\mathbf{a}}_{N 2} \\
\overline{\mathbf{a}}_{R 1} & \bar{a}_{R 2}
\end{array}\right]\left[\begin{array}{c}
\mathbf{z}_{N} \\
z_{R}
\end{array}\right]+\left[\begin{array}{c}
\mathbf{0} \\
\bar{b}_{r}
\end{array}\right] u+\left[\begin{array}{c}
\overline{\mathbf{v}}_{N} \\
0
\end{array}\right] r+\left[\begin{array}{c}
\overline{\mathbf{d}}_{N} \\
0
\end{array}\right] \xi_{t} \\
& \Leftrightarrow \begin{array}{c}
\dot{\mathbf{z}}_{N}=\overline{\mathbf{A}}_{N 1} \mathbf{z}_{N}+\overline{\mathbf{a}}_{N 2} z_{R}+\overline{\mathbf{v}}_{N} r+\overline{\mathbf{d}}_{N} \xi_{t} \\
\dot{z}_{R}=\overline{\mathbf{a}}_{R 1} \mathbf{z}_{N}+\bar{a}_{R 2} z_{R}+\bar{b}_{r} u
\end{array} \\
& f_{c}=\left[\begin{array}{ll}
\overline{\mathbf{c}}_{N} & 0
\end{array}\right]\left[\begin{array}{c}
\mathbf{z}_{N} \\
z_{\mathrm{R}}
\end{array}\right]+d \xi_{t}=\overline{\mathbf{c}}_{N} \mathbf{z}_{N}+d \xi_{t} \\
& \text { where } \quad \mathbf{z}_{N} \in \mathfrak{R}^{6 \times 1}, \quad z_{R} \in R^{1}, \overline{\mathbf{A}}_{N 1} \in \mathfrak{R}^{6 \times 6}, \overline{\mathbf{a}}_{N 2} \in \mathfrak{R}^{6 \times 1}, \overline{\mathbf{a}}_{R 1} \in \mathfrak{R}^{1 \times 6}, \bar{a}_{R 2} \in R^{1}, \\
& \overline{\mathbf{v}}_{N} \in \mathfrak{R}^{6 \times 1}, \bar{b}_{r} \in R^{1}, \overline{\mathbf{d}}_{N} \in \mathfrak{R}^{6 \times 1}, \overline{\mathbf{c}}_{N} \in \mathfrak{R}^{1 \times 6},
\end{aligned}
$$

The coefficient of the switching function is also partitioned corresponding to the subsystems as

$$
\sigma=\left[\begin{array}{ll}
\mathbf{S}_{N} & 1
\end{array}\right] \mathbf{z}=\left[\begin{array}{ll}
\mathbf{S}_{N} & 1
\end{array}\right]\left[\begin{array}{c}
\mathbf{z}_{\mathrm{N}} \\
Z_{\mathrm{R}}
\end{array}\right]
$$

where the last element in $\mathbf{S}$ is assumed to be 1 without any loss of generality. Since $\sigma=0$ and the system order is reduced by one during ideal sliding mode, the dynamics of the sliding mode is represented by Eq. (14) and $\sigma=0$ with Eq.(17) instead of the range space dynamics Eq.(15) as

$$
Z_{R}=-\mathbf{S}_{N} \mathbf{z}_{N}
$$

One of the advantages of this form is that $z_{R}$ can be thought of as virtual linear state feedback control for the Null space dynamics (14), and thus the coefficient of the switching function, $\mathbf{S}_{N}$, can be determined by linear control theory as a feedback gain vector. In this paper, therefore, we divide the Null space dynamics further into two subsystems. To this end, the state vector and the other matrices in Eqs. (14), (18) are partitioned as

$$
\begin{aligned}
& \mathbf{z}_{N}=\left[\begin{array}{c}
z_{N 1} \\
\mathbf{z}_{N 2}
\end{array}\right], \overline{\mathbf{a}}_{N 2}=\left[\begin{array}{c}
0 \\
\hat{\mathbf{a}}_{N 2}
\end{array}\right], \overline{\mathbf{A}}_{N 1}=\left[\begin{array}{cc}
0 & -\hat{\mathbf{c}}_{N} \\
0 & \hat{\mathbf{A}}_{N 1}
\end{array}\right], \overline{\mathbf{c}}_{N}=\left[\begin{array}{ll}
0 & \hat{\mathbf{c}}_{N}
\end{array}\right], \mathbf{S}_{N}=\left[\begin{array}{ll}
S_{N 1} & \mathbf{S}_{N 2}
\end{array}\right] \\
& \text { where } Z_{N 1} \in \mathfrak{R}^{1}, \quad \mathbf{z}_{N 2} \in \mathfrak{R}^{5 \times 1}, \hat{\mathbf{a}}_{N 2}=\mathfrak{R}^{5 \times 1}, \hat{\mathbf{c}}_{N} \in \mathfrak{R}^{1 \times 5}, \hat{\mathbf{A}}_{N 1} \in \mathfrak{R}^{5 \times 5}, \quad S_{N 1} \in \mathfrak{R}^{1}, \mathbf{S}_{N 2} \in \mathfrak{R}^{1 \times 5}
\end{aligned}
$$

where we use $\hat{\mathbf{c}}_{N}$ in $\overline{\mathbf{A}}_{N 1}$ noting that the first row in $\overline{\mathbf{A}}$ given by Eq. (10) originally contains the coefficient of the output in Eq. (7). Using these matrices and assuming $\xi_{t}=0$ to get a nominal model for controller design, Eq. (14) is rewritten as

$$
\begin{aligned}
& \dot{z}_{N 1}=r-\hat{\mathbf{c}}_{N} \mathbf{z}_{N 2}=r-f_{c} \\
& \dot{\mathbf{z}}_{N 2}=\hat{\mathbf{A}}_{N 1} \mathbf{z}_{N 2}+\hat{\mathbf{a}}_{N 2} Z_{R}
\end{aligned}
$$

where we use the fact

$$
f_{c}=\mathbf{c X}=\overline{\mathbf{c}}_{N} \mathbf{z}_{N}=\hat{\mathbf{c}}_{N} \mathbf{z}_{N 2}
$$

which is easily derived from Eq. (16) under the assumption $\xi_{t}=0$. In a similar way the virtual control Eq. (18) can be rewritten as 


$$
Z_{R}=-\mathbf{S}_{N} \mathbf{z}_{N}=-S_{N 1} z_{N 1}-\mathbf{S}_{N 2} \mathbf{z}_{N 2}=-S_{N 1} \int\left(r-f_{c}\right) d \tau-\mathbf{S}_{N 2} \mathbf{z}_{N 2}
$$

Equations (20) to (23) are represented by the block diagram as shown in Fig. 5, from which it turns out that the coefficient of the switching function can be thought of as a feedback gain vector for the virtual plant Eq. (21) with an integral compensator by Eq. (20) or (23).

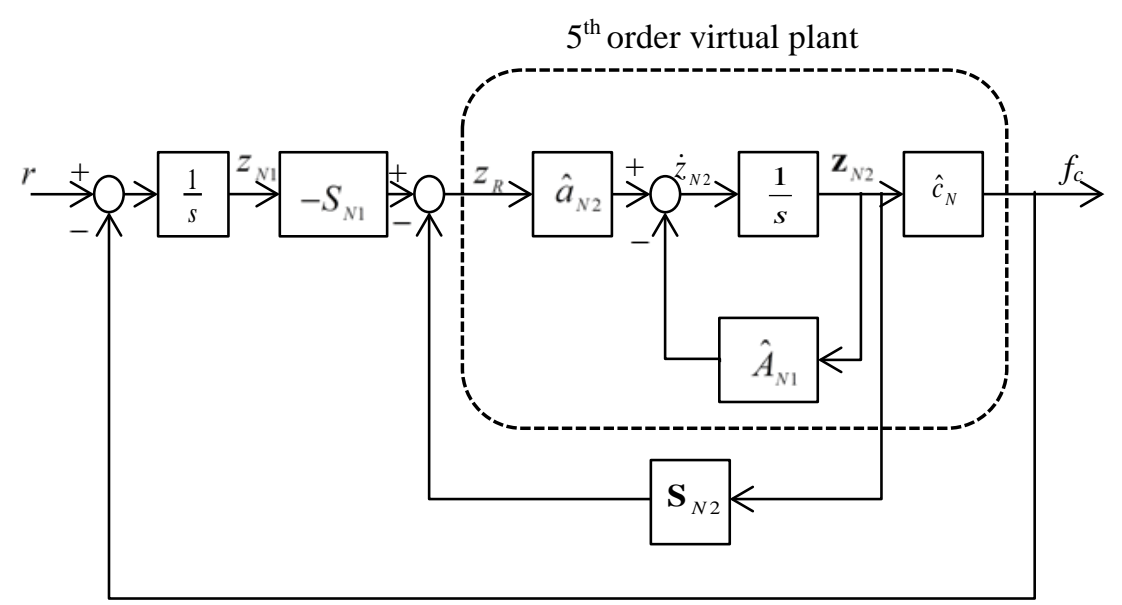

Fig. 5 During the ideal sliding mode induced by the control law Eq. (12), its dynamics can be thought of as a virtual linear servo system with reduced order as shown here. This observation leads to employing linear design methodologies for the switching functions in the sliding mode controller.

Next we will apply the design method of optimal linear tracking systems proposed by Takeda and Kitamori to determine an optimal switching function. There are two features in their design approach. One of them is to use the control input instead of the output of the integral compensator as a state variable, i.e. $Z_{R}$ instead of $Z_{N 1}$ in the equivalent system shown in Fig. 5. Another one is to use a deviation system from the steady state under the assumption the reference signal is a step function or constant. As a result, the tracking error, $r-f_{c}$, can be thought of as the system output, and it can be weighted in the quadratic performance function as shown below.

Following their method with Eqs. (20) to (23), we can derive the deviation system from the steady state for the virtual linear servo system as follows:

$\left[\begin{array}{c}\dot{\mathbf{z}}_{N 2 e} \\ \dot{\mathbf{Z}}_{\operatorname{Re}}\end{array}\right]=\left[\begin{array}{cc}\hat{\mathbf{A}}_{N 1} & \hat{\mathbf{a}}_{N 2} \\ \mathbf{0} & \mathbf{0}\end{array}\right]\left[\begin{array}{c}\mathbf{z}_{N 2 e} \\ Z_{\operatorname{Re}}\end{array}\right]+\left[\begin{array}{l}\mathbf{0} \\ 1\end{array}\right] \omega$

where $\quad \mathbf{z}_{N 2 e}=\mathbf{z}_{N 2}-\mathbf{z}_{N 2}(\infty), z_{\mathrm{Re}}=z_{\mathrm{R}}-z_{\mathrm{R}}(\infty)$, and

$\omega=\dot{Z}_{\mathrm{Re}}=-\mathbf{f}_{e}\left[\begin{array}{c}\mathbf{z}_{N 2 e} \\ Z_{\mathrm{Re}}\end{array}\right]$

where

$$
\begin{aligned}
& \mathbf{f}_{e}=\left[\begin{array}{ll}
\mathbf{S}_{N 2} & -S_{N 1}
\end{array}\right]\left[\begin{array}{cc}
\hat{\mathbf{A}}_{N 1} & \hat{\mathbf{a}}_{N 2} \\
\hat{\mathbf{c}}_{N} & 0
\end{array}\right] \\
& e=r-f_{c}=\left[\begin{array}{ll}
\hat{\mathbf{c}}_{N} & 0
\end{array}\right]\left[\begin{array}{c}
\mathbf{z}_{N 2 e} \\
Z_{\mathrm{Re}}
\end{array}\right]
\end{aligned}
$$

Equation (24) can be thought of as a plant to be controlled with the control input $\omega$ which is linear state feedback Eq. (25), and Eq. (27) can be thought of as the output equation. Because the feedback gain $\mathbf{f}_{e}$ includes the coefficient vector of the switching function in Eq. (26), it is given by

$$
\left[\begin{array}{ll}
\mathbf{S}_{N 2} & -S_{N 1}
\end{array}\right]=\left[\begin{array}{cc}
\hat{\mathbf{A}}_{N 1} & \hat{\mathbf{a}}_{N 2} \\
\hat{\mathbf{c}}_{N} & 0
\end{array}\right] \mathbf{f}_{e}^{-1}
$$

once $\mathbf{f}_{e}$ is obtained. The matrix on the right-hand side in Eq. (28) is non-singular because the virtual plant Eqs. (21), 
(22) does not have any zeros at the origin as the original plant Eqs. (4), (7) (cf. Eq. (8) to obtain zeros in the state space form). Finally, applying the LQR technique with the performance index

$$
J=\int_{0}^{\infty}\left\{q e^{2}(t)+\omega^{2}\right\} d t
$$

the optimal feedback gain $\mathbf{f}_{e}$ is obtained by solving the standard Riccati equation. It should be noted here that the tracking error is directly weighted by $q$, and the virtual control input is $\omega=\dot{z}_{\mathrm{Re}}=\ddot{x}_{f 2}$. That is, the optimal control obtained under this performance index achieves the trade-off between the tracking error and the acceleration of the lower frame. However, evaluating the acceleration of the lower frame means to penalize the use of control effort implicitly, because the equation of motion (3) implies that it is highly depend on the control force $f_{a}$.

\subsection{Analysis of the optimal servo system}

In this subsection, we will apply the SRL (Symmetric Root Locus) method (Franklin et al., 2010), which gives the locus of the optimal poles with respect to the parameter $q$ under the performance index (29), to analyze the optimal servo system designed in the previous subsection. The optimal poles are the stable roots in the left half plane of the SRL equation

$$
1+\rho G_{N}(-s) G_{N}(s)=0
$$

where $G_{N}(s)$ is the transfer function of the virtual control input $\omega$ to the tracking error $e$,

$$
G_{N}(s)=\left[\begin{array}{ll}
\hat{\mathbf{c}}_{N} & 0
\end{array}\right]\left(s \mathbf{I}-\left[\begin{array}{cc}
\hat{\mathbf{A}}_{N 1} & \hat{\mathbf{a}}_{N 2} \\
\mathbf{0} & \mathbf{0}
\end{array}\right]\right)^{-1}\left[\begin{array}{l}
\mathbf{0} \\
1
\end{array}\right]
$$

The symmetric root locus obtained for $q=10^{-2} \sim 10^{5}$ is shown in Fig. 6, where a portion around the origin in the left figure is zoomed in the right figure.

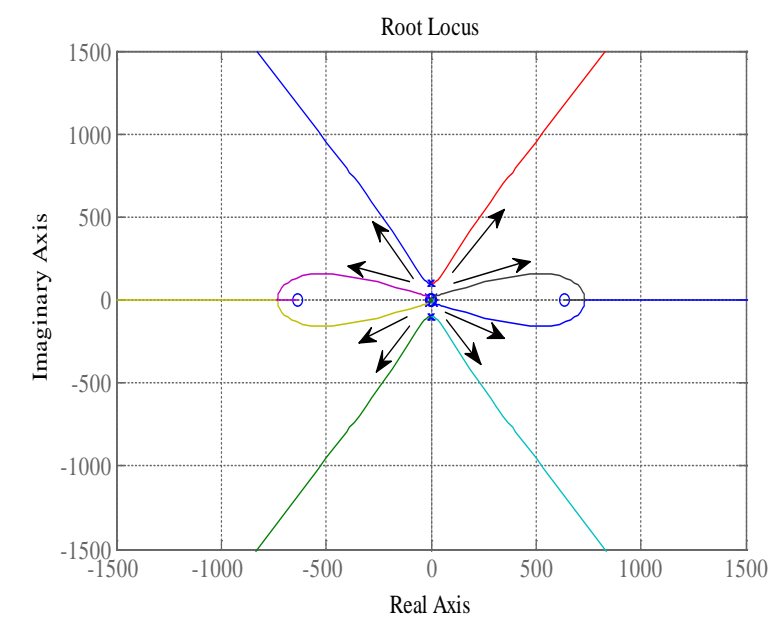

(a) overall

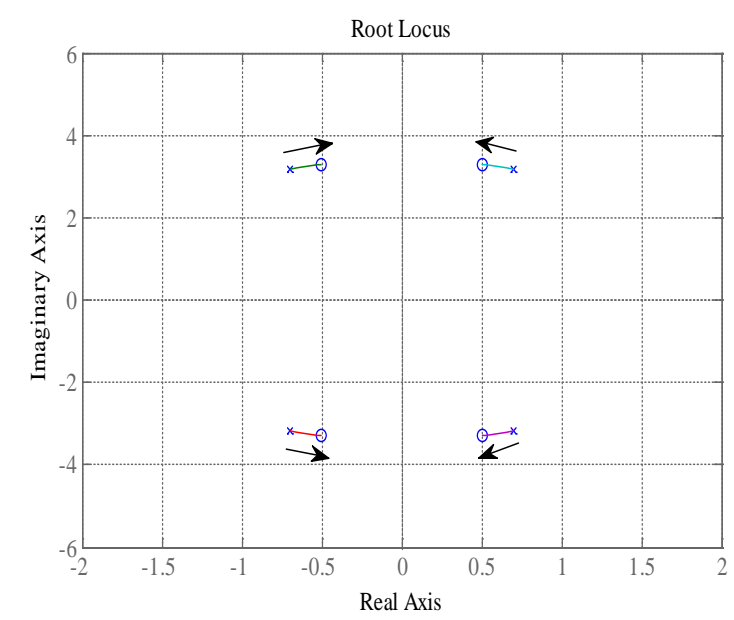

(b) around the origin

Fig. 6 Root locus of optimal sliding mode poles. As the weighting factor with respect to the tracking error in the performance index (29) increases, a pair of poles asymptotically approach to the zeros which are the exactly same as the poles of the nominal catenary subsystem Eq. (5)

As shown in this figure, the stable roots converge to the zeros of $G_{N}(s)$ as $\rho$ increases, which is the same property as normal root locus. Noting that these zeros are identical to those of the virtual plant in Fig. 5, they must satisfy the determinant equation with respect to $s$ 


$$
\left|\begin{array}{cc}
\hat{\mathbf{A}}_{N 1}-s \mathbf{I} & \hat{\mathbf{a}}_{N 2} \\
\hat{\mathbf{c}}_{N} & 0
\end{array}\right|=0
$$

On the other hand, the determinant equation for the zeros of the original plant Eq. (8) can be rewritten by expanding the determinant with respect to the last column as

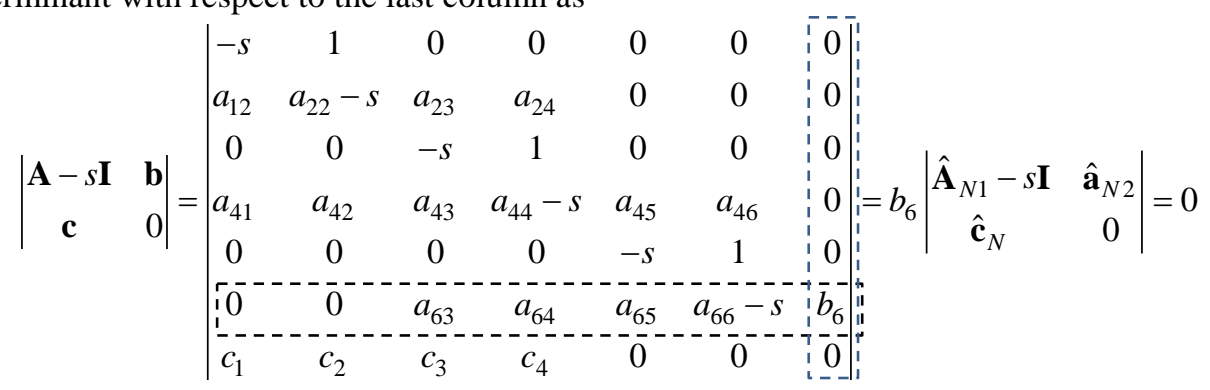

where $a_{i j}$ is an element of $\mathbf{A}, b_{i}$ is an element of $\mathbf{b}$, and $c_{i}$ is an element of $\mathbf{c}$.

It can be seen from Eqs. (32), (33) that the optimal poles converge to the zeros of the original plant Eq. (9). As mentioned below Eq. (9), the complex zeros near the origin are identical to the poles of the nominal catenary subsystem Eq. (5). This asymptotic property reveals that two optimal poles converge to the catenary poles as $\rho$ increases so that pole-zeros cancellations are approximately brought about. This analytical result is very sound from a physical point of view, because the pantograph should follow the catenary motion to reduce the contact force variation.

\subsection{Sliding mode observer}

In this subsection, we design a sliding mode observer based on a design method of modified Walcott-Zack observer (Shtessel et al. 2014). In general servo systems, the controlled variables are used for the observer. However, the contact force is not appropriate for the observer, because it is directly influenced by the uncertainty as seen from Eq. (16). Taking account of robustness against the uncertainty and the existence of sliding mode, therefore, we use two measurements, the velocity of the contact wire/shoe $\dot{x}_{t s}$ and the displacement of the lower frame $x_{f 2}$, for the observer. To simplify the design procedure, changing the order of the state variables in the state vector, an alternative state vector is defined as

$$
\tilde{\mathbf{x}} \triangleq\left[\begin{array}{llllll}
x_{t s} & x_{f 1} & \dot{x}_{f 1} & \dot{x}_{f 2} & x_{f 2} & \dot{x}_{t s}
\end{array}\right]^{T}
$$

so that the last two elements are the measurements. Using this new state vector, the plant to be controlled Eq. (4) can be rewritten and partitioned as

$$
\begin{aligned}
\dot{\tilde{\mathbf{x}}} & =\tilde{\mathbf{A}} \tilde{\mathbf{x}}+\tilde{\mathbf{b}} u+\tilde{\mathbf{d}}_{t} \xi_{t} \\
& \triangleq\left[\begin{array}{cc}
\tilde{\mathbf{A}}_{11} & \tilde{\mathbf{A}}_{12} \\
\tilde{\mathbf{a}}_{211} & \tilde{\mathbf{A}}_{22} \\
\tilde{\mathbf{a}}_{212} &
\end{array}\right] \tilde{\mathbf{x}}+\tilde{\mathbf{b}} f_{a}+\tilde{\mathbf{d}}_{t} \xi_{t}, \tilde{\mathbf{A}}_{11} \in \mathfrak{R}^{4 \times 4}, \tilde{\mathbf{A}}_{12} \in \mathfrak{R}^{4 \times 2}, \tilde{\mathbf{a}}_{211} \in \mathfrak{R}^{1 \times 4}, \tilde{\mathbf{a}}_{212} \in \mathfrak{R}^{1 \times 4}, \tilde{\mathbf{A}}_{22} \in \mathfrak{R}^{2 \times 2}
\end{aligned}
$$

The block matrices will be used later. The output equation with respect to the measurements is represented by

$$
\tilde{\mathbf{y}}=\left[\begin{array}{llllll}
0 & 0 & 0 & 0 & 1 & 0 \\
0 & 0 & 0 & 0 & 0 & 1
\end{array}\right] \tilde{\mathbf{x}}=\mathbf{H} \tilde{\mathbf{x}}
$$

The sliding mode observer is designed as 


$$
\begin{aligned}
& \dot{\tilde{\tilde{\mathbf{x}}}}=\tilde{\mathbf{A}} \hat{\tilde{\mathbf{x}}}+\tilde{\mathbf{b}} u-\mathbf{G}_{l} \tilde{\mathbf{e}}_{y}+\mathbf{G}_{n} \mathbf{v} \\
& \text { where } \tilde{\mathbf{e}}_{y}=\hat{\tilde{\mathbf{y}}}-\tilde{\mathbf{y}}, \hat{\tilde{\mathbf{y}}}=\mathbf{H} \hat{\tilde{\mathbf{x}}}, \mathbf{v}=-\rho \frac{\tilde{\mathbf{e}}_{y}}{\left\|\tilde{\mathbf{e}}_{y}\right\|}, \mathbf{G}_{l} \in \mathfrak{R}^{6 \times 2}, \mathbf{G}_{n} \in \mathfrak{R}^{6 \times 2}
\end{aligned}
$$

In this observer, the last term on the right-hand side is a discontinuous function to bring about the sliding mode, and the estimation error with respect to the measurements, $\tilde{\mathbf{e}}_{y}$, is the switching function, i.e. during sliding mode $\tilde{\mathbf{e}}_{y}=\dot{\tilde{\mathbf{e}}}_{y}=0$. Defining the estimation error as

$$
\tilde{\mathbf{e}} \triangleq \hat{\tilde{\mathbf{x}}}-\tilde{\mathbf{x}}=\left[\begin{array}{c}
\tilde{\mathbf{e}}_{1} \\
\tilde{\mathbf{e}}_{y}
\end{array}\right]
$$

it follows from Eqs. (35) and (37) that the estimation error dynamics is given by

$$
\dot{\tilde{\mathbf{e}}}=\tilde{\mathbf{A}} \tilde{\mathbf{e}}-\mathbf{G}_{l} \tilde{\mathbf{e}}_{y}+\mathbf{G}_{n} \mathbf{v}-\tilde{\mathbf{d}} \xi_{t}, \text { where } \tilde{\mathbf{d}}=\left[\begin{array}{c}
\mathbf{0}_{5} \\
d
\end{array}\right] \triangleq\left[\begin{array}{l}
\mathbf{0}_{4} \\
\mathbf{d}_{2}
\end{array}\right], \mathbf{d}_{2} \in \mathfrak{R}^{2 \times 1}
$$

As seen from this equation, one of the points to determine the design parameter $\mathbf{G}_{n}$ is that if $\tilde{\mathbf{d}}$ belongs to the range space of $\mathbf{G}_{n}$, i.e. the matching condition holds, then the estimation error dynamics during sliding mode is insensitive to the uncertainty. Although this requirement can be easily fulfilled, there is another obstacle such as the existence of sliding mode, which is highly depend not only on $\mathbf{G}_{n}$ but also on the linear gain $\mathbf{G}_{l}$ as another design parameter. To our best knowledge, there is no systematic ways of designing these parameters, but the method proposed by Edwards (Shtessel et al. 2014) seems like better than the others. Taking the matching condition into account, firstly $\mathbf{G}_{n}$ is parameterized as

$$
\mathbf{G}_{n}=\left[\begin{array}{l}
-\mathbf{L} \\
\mathbf{I}_{2}
\end{array}\right] \mathbf{P}_{0}^{-1} \text {, where } \mathbf{L}=\left[\begin{array}{ll}
\mathbf{l}_{0} & \mathbf{0}
\end{array}\right] \in \mathfrak{R}^{4 \times 2}, \mathbf{l}_{0} \in \mathfrak{R}^{4 \times 1}, \mathbf{P}_{0} \in \mathfrak{R}^{2 \times 2}
$$

It should be noted that the last column in $\mathbf{G}_{n}$ is taken for $\tilde{\mathbf{d}}$ to belong to the range space of $\mathbf{G}_{n}$.

Next, to change the error dynamics Eq. (39) into the regular form, using the linear transformation as

$$
\tilde{\mathbf{e}} \mapsto \mathbf{T}_{L} \tilde{\mathbf{e}}=\tilde{\mathbf{e}}^{\prime}=\left[\begin{array}{c}
\tilde{\mathbf{e}}_{1}^{\prime} \\
\tilde{\mathbf{e}}_{2}
\end{array}\right] \text {, where } \quad \mathbf{T}_{L}=\left[\begin{array}{cc}
\mathbf{I}_{4} & \mathbf{L} \\
0 & \mathbf{I}_{2}
\end{array}\right]
$$

it follows the null space dynamics and range space dynamics,

$$
\begin{aligned}
& \dot{\tilde{\mathbf{e}}}_{1}^{\prime}=\left(\tilde{\mathbf{A}}_{11}+\mathbf{l}_{0} \tilde{\mathbf{a}}_{211}\right) \tilde{\mathbf{e}}_{1}^{\prime}+\left(\tilde{\mathbf{A}}_{12}^{\prime}-\mathbf{G}_{l 1}^{\prime}\right) \tilde{\mathbf{e}}_{y} \\
& \dot{\tilde{\mathbf{e}}}_{y}=\tilde{\mathbf{A}}_{21}^{\prime} \tilde{\mathbf{e}}_{1}^{\prime}+\left(\tilde{\mathbf{A}}_{22}^{\prime}-\mathbf{G}_{l 2}^{\prime}\right) \tilde{\mathbf{e}}_{y}+\mathbf{P}_{0}^{-1} \mathbf{v}-\mathbf{d}_{2} \xi_{t}
\end{aligned}
$$

respectively, where the superscript(') means that its matrix or vector is transformed by $\mathbf{T}_{L}$ such as

$$
\mathbf{T}_{L} \tilde{\mathbf{A}} \mathbf{T}_{L}^{-1} \triangleq\left[\begin{array}{cc}
\tilde{\mathbf{A}}_{11}^{\prime} & \tilde{\mathbf{A}}_{12}^{\prime} \\
\tilde{\mathbf{A}}_{21}^{\prime} & \tilde{\mathbf{A}}_{22}^{\prime}
\end{array}\right], \quad \mathbf{T}_{L} \mathbf{G}_{l} \triangleq\left[\begin{array}{c}
\mathbf{G}_{l 1}^{\prime} \\
\mathbf{G}_{l 2}^{\prime}
\end{array}\right]
$$

The null space dynamics (42) is insensitive to the uncertainty because of the matching condition, and thus the estimation error dynamics during sliding mode, $\tilde{\mathbf{e}}_{y}=\dot{\tilde{\mathbf{e}}}_{y}=0$, is governed by

$$
\dot{\tilde{\mathbf{e}}}_{1}^{\prime}=\left(\tilde{\mathbf{A}}_{11}+\mathbf{l}_{0} \tilde{\mathbf{A}}_{211}\right) \tilde{\mathbf{e}}_{1}^{\prime}
$$


which can be determined by the design parameter $\mathbf{l}_{0}$. The other design parameters must be determined to guarantee the existence of sliding mode.

\section{Numerical simulation}

In order to investigate the performance of the proposed servo system, numerical simulation has been carried out. The parameter values of the pantograph-catenary system are shown in Table 1, where the parameter values of the pantograph are the estimates by identification experiments, and those of the catenary system are determined based on some references ( Kobayashi et al., 2014). When the train speed is $360 \mathrm{~km} / \mathrm{h}$ and the span length of each cantilever is about $50 \mathrm{~m}$, the catenary equivalent stiffness can be assumed to change periodically with $2 \mathrm{~Hz}$ as shown in Fig. 7 (Usuda and Ikeda, 2014).

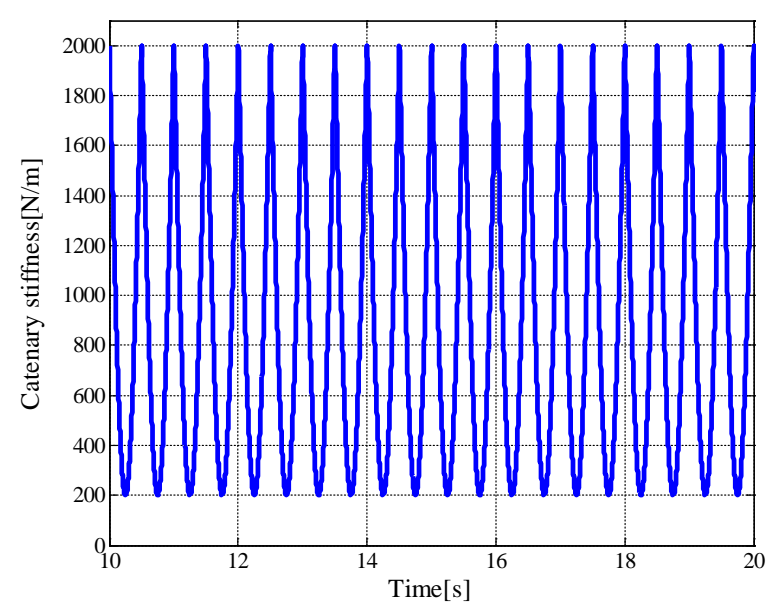

Fig. 7 Perturbation with respect to the equivalent stiffness of catenary due to the cantilevers under the assumption that the train speed is $360 \mathrm{~km} / \mathrm{h}$.

Because the proposed servo system achieved a good performance as expected, we carried out some simulations in more realistic situation. That is, although we neglected the actuator dynamics when designing the controller and observer, we inserted the following dynamic model of the pneumatic actuator

$$
G(s)=\frac{1}{0.013 s+1} e^{-0.002 s}
$$

which had been obtained by some experiments. All the simulation results to be shown later were obtained by using this actuator model except for Fig. 9. Furthermore, it was assumed that three measurements for the controller and observer were corrupted by white Gaussian noises whose maximum magnitude are as follows:

contact force: $\pm 4 \mathrm{~N}$, displacement of the lower frame: $\pm 1 \times 10^{-4} \mathrm{~m}$, velocity of the contact wire/shoe head: $\pm 1 \times 10^{-4} \mathrm{~m} / \mathrm{s}$.

The design parameters in the controller and observer were determined taking account of this situation. In addition, to reduce chattering due to the relay component of the control law, we used a saturation function with boundary layer instead of the relay. The main design parameters in the controller are as follows:

weighting factor : $q=1$

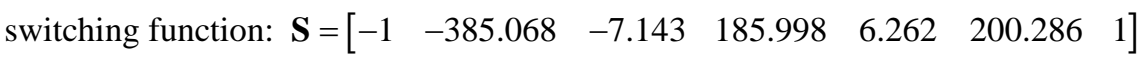

relay gain: $\rho=100$ and boundary layer: \pm 0.05

With these parameters, the poles and zeros in the transfer function from the reference signal to the contact force during sliding mode are obtained as follows:

$$
\text { Zeros }=\left[\begin{array}{ll}
-633.33-0.5 \pm 3.28 j
\end{array}\right]
$$




$$
\text { Poles }=\left[\begin{array}{lll}
-29.89 \pm 119.69 j & -75.54 \pm 46.09 j & -0.5 \pm 3.28 j
\end{array}\right]
$$

It should be noted that a pair of poles cancel out a pair of zeros at $-0.5 \pm 3.28 j$ which is the mode of the catenary subsystem (9) as mentioned in section 2 and subsection 3.2.

Taking these poles into account, the observer poles during sliding mode were determined as $-70 \pm 10 i,-50 \pm 10 i$ after some trial and errors. In the rest of simulation, the reference signal is given as

$$
r(t)=\left\{\begin{array}{cc}
54 & \text { for } 0 \leq t \leq 11 \\
64 & \text { thereafter }
\end{array}\right.
$$

to make sure of not only the steady state response but also the transient response.

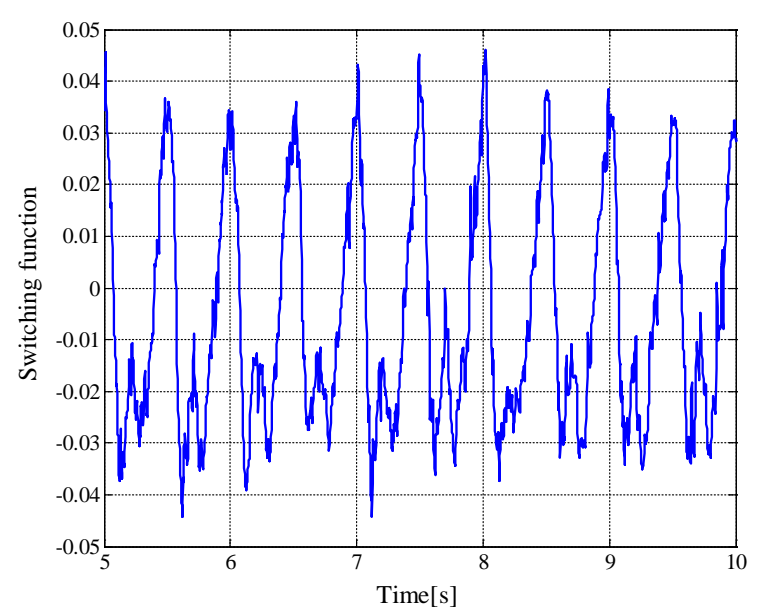

(a) In controller

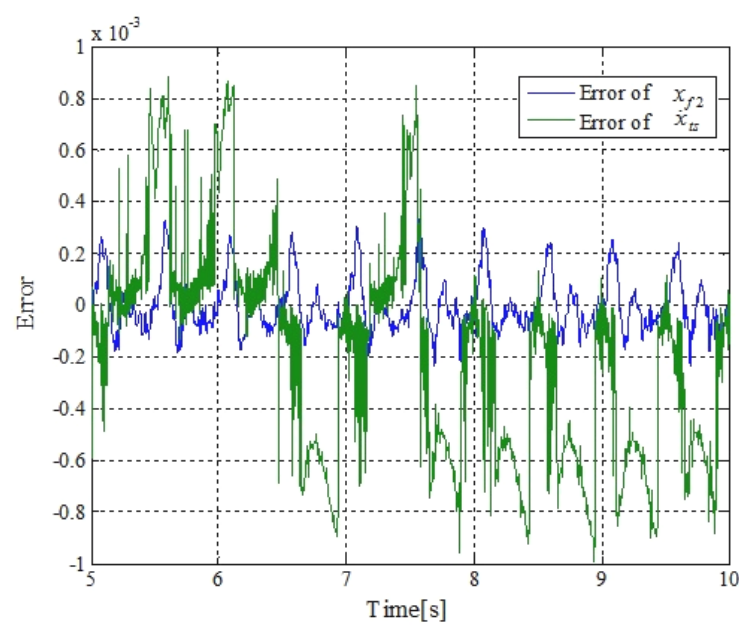

(b) In observer

Fig. 8 Switching functions in steady state. The switching functions in the observer in Fig (b) are the same as the estimation errors for $x_{f 2}, \dot{x}_{t s}$. It can be thought that sliding modes take place approximately, because the values of these functions are quite small.

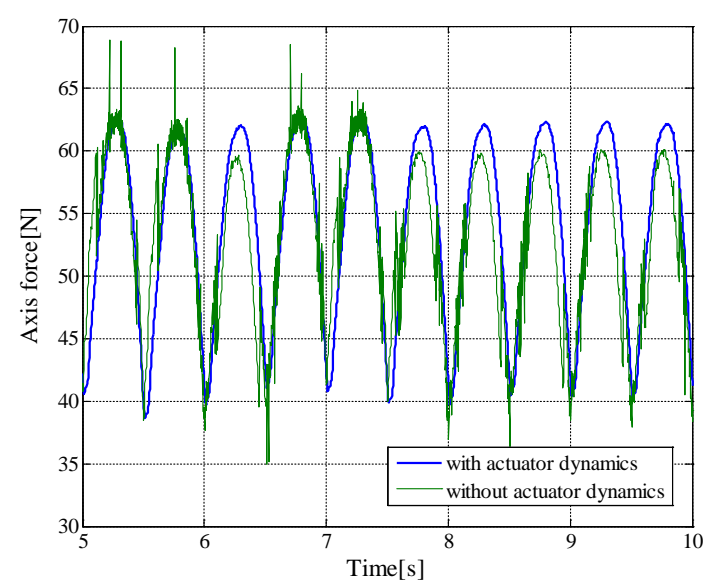

Fig. 9 Comparison of the control inputs. Because the actuator dynamics was not taken into account in the nominal model for controller design, its effect on the control performance was investigated especially by comparing the control inputs. "Without actuator dynamics" in the legend means that the simulation was carried out without the actuator dynamics, or equivalently the dynamics was ignored. It can be seen from this figure that the actuator dynamics does not affect the control input so much. 


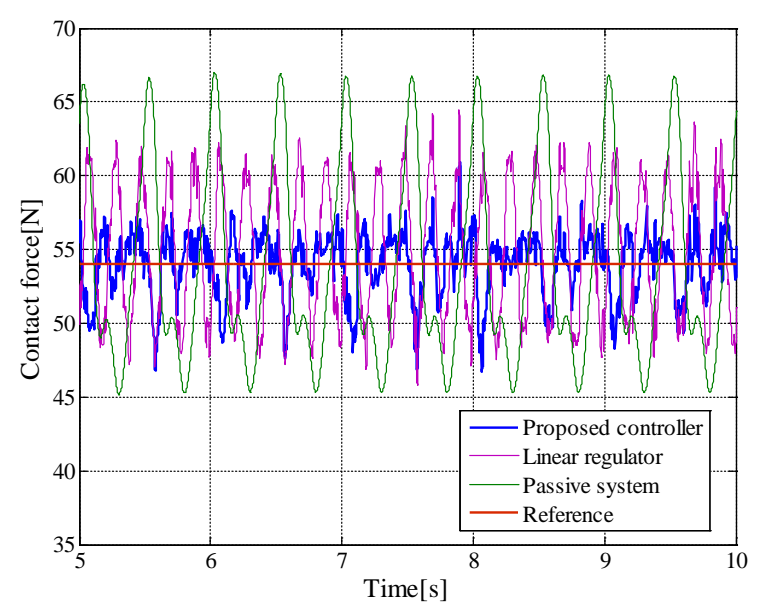

(a) Contact force

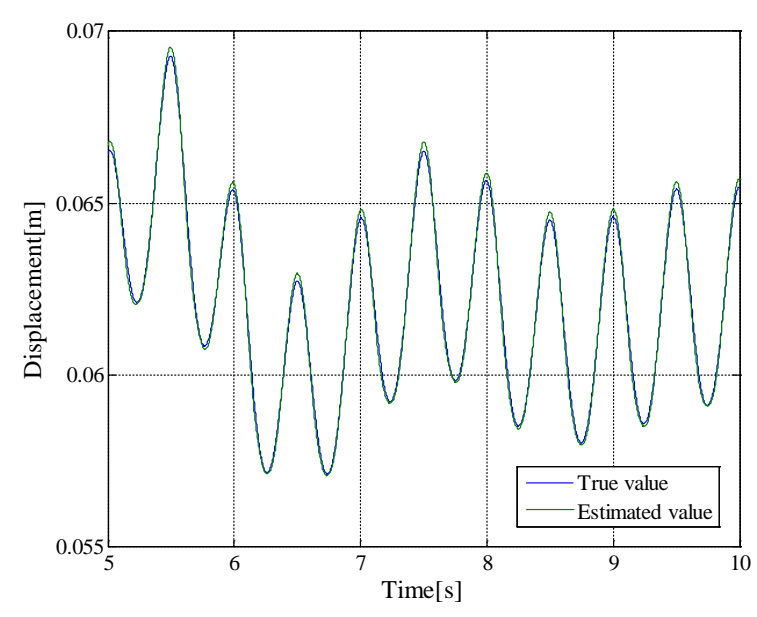

(b) Catenary displacement

Fig. 10 Steady state response. Figure (a) shows the contact force in three different systems: proposed one with the actuator dynamics, linear regulator with the actuator dynamics, and passive one. The linear regulator was designed based on optimal control theory together with a linear observer. Figure (b) shows the true value and estimated one of the catenary displacement for the proposed controller.

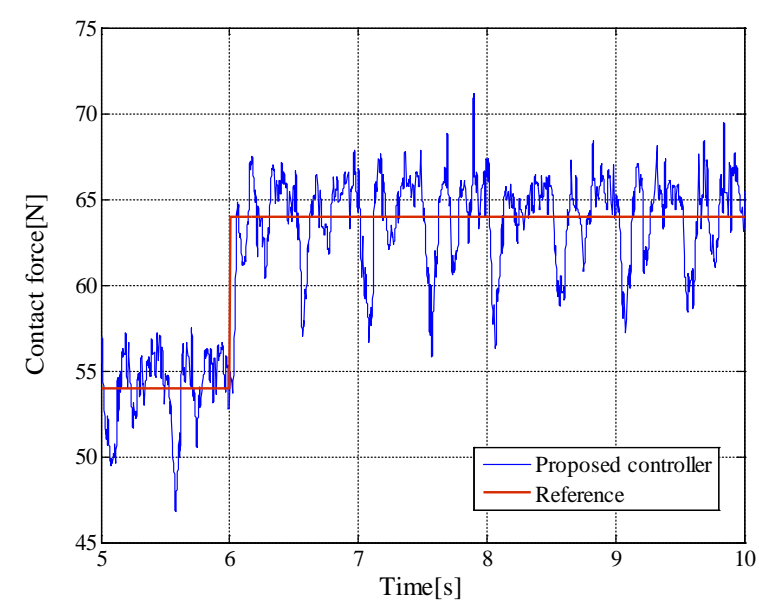

(a) Contact force

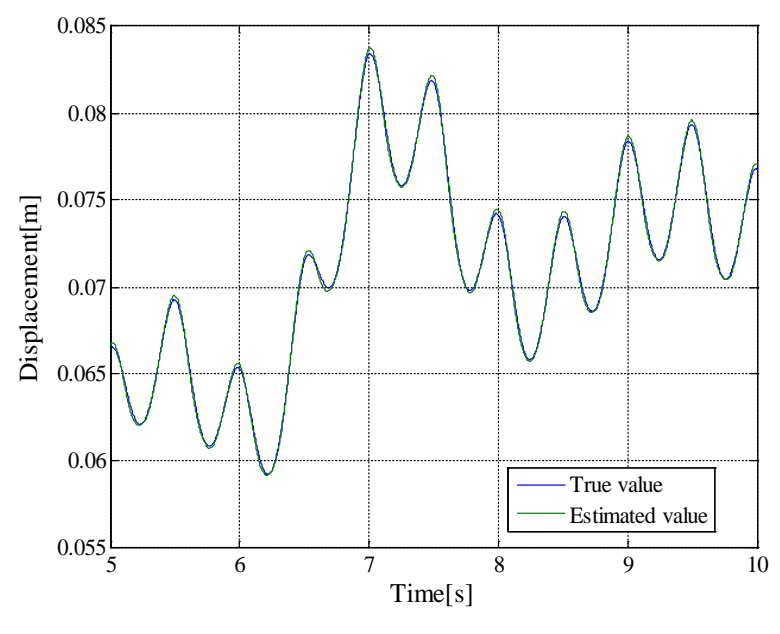

(b) Catenary displacement

Fig. 11 Step response of the proposed servo system with the actuator dynamics.

Figure 8 shows the switching functions in the controller and observer in steady state. It can be seen from Fig. 8(a) that the switching function in the controller stays within the small boundary layer, which can prove that quasi-sliding mode takes place in spite of the existence of the actuator dynamics (46). It can be seen from Fig. 8(b) that the estimation errors, $\mathbf{e}_{y}$, which is the switching function vector as well, contains high frequency components due to the sensor noise but quite small, yielding the sliding mode.

Because the actuator dynamics was not taken into account in the plant model for controller design, its effect on the control performance was investigated. Figure 9 shows the two control inputs, one is for the plant model with the actuator dynamics and the other is for the plant model without the actuator dynamics (ignoring the actuator dynamics). They can be considered to be almost the same, which means that the controller demands appropriate control force for the actuator dynamics such as bandwidth. The steady state response is shown in Fig. 10. Figure 10(a) shows the contact force in three different systems: (1) proposed one with the actuator dynamics, (2) linear regulator with the actuator dynamics, (3) passive one. The linear regulator was designed based on 
optimal control theory together with a linear observer. It can be seen from this figure that the proposed controller regulates the contact force much better than the others by keeping the variation of the catenary displacement very small with the highly accurate estimates by the observer, as shown in Fig. 10(b).

Figure 11 shows the step response of the catenary displacement and contact force, from which it can be seen that the transient response is also good with small rise time and no overshoot except for that due to the steady variation.

\section{Conclusion}

In this paper, we have proposed a sliding mode controller using optimal servo control theory and sliding mode observer, taking account of the flexibility of the articulated frame in the actual pantograph. It has been pointed out through our analysis of the plant and the closed-loop system using SRL (symmetric root locus) technique that pole-zero cancellations play an important role to control the contact force. A physical interpretation of the pole-zero cancellations is that the pantograph head should follow the catenary motion to reduce the variation of the contact force. The simulation results have demonstrated the good performance and robustness of the proposed control system in the presence of variation of the catenary stiffness.

Although the measurements for the observer were assumed to be the velocity of the contact-wire/shoe and the displacement of the lower frame, other choices should be investigated taking more practical situations such as noise level into account. Much more rigorous analysis of the closed-loop stability dealing the plant as a time-varying system is still under investigation (Myat Thiri Ko, et al., 2016c).

\section{Acknowledgment}

This research was partially supported by Railway Technical Research Institute. The authors would like to thank Dr. Y. Yamashita, Dr. T. Usuda and Dr. S. Kobayashi of the institute.

\section{References}

Arnold, M. and Simeon, B., Pantograph and catenary dynamics, A benchmark problem and its numerical solution Applied Numerical Mathematics (2000) 34, pp.345-362.

Allotta, B., Pisano, A., Pugi, L. and Usai, E., VSC of a servo-actuated ATR 90-type pantograph, Proceedings of the $44^{\text {th }}$ IEEE Conference on Decision and Control, and the European Control Conference (2005), pp.590-595.

Chater, E., Ghani, D., Giri, F., Rachid, A., Chaoui, F.Z. and Haloua, M., Output feedback control of pantograph-catenary system 5th IFAC International Workshop on Periodic Control Systems, Vol. 5, No. 1 (2013), pp.131-136.

Edwards, C. and Spurgeon, S., Sliding mode control-Theory and applications (1998), Taylor and Francis.

Franklin, G.F., Powell, J.D. and Emami-Naeini, A., Feedback Control of Dynamic Systems (2010), pp.475-483 Pearson.

Kobayashi, S., Usuda, T. and Ikeda, M., Application of HILS technique to pantograph-catenary system, RTRI Report vol. 28, No.12 (2014), pp.5-10 (in Japanese).

Makino, T., Yoshida, K., Seto, S. and Makino, K., Running test on current collector with contact force controller for high-speed railways, JSME International Journal Series, Vol. C 40, No. 4 (1997), pp.671-680.

Myat Thiri Ko, Yokoyama, M., Yamashita, Y,. Kobayashi, S. and Usuda, T., Contact force control of an active pantograph for high speed trains, Proceedings of the $13^{\text {th }}$ International Conference on Motion \& Vibration Control $/ 12^{\text {th }}$ Recent Advances in Structural Dynamics (MOVIC/RASD) (2016a).

Myat Thiri Ko, Yokoyama, M., Nagayoshi, S., Robust contact force control of an active pantograph via sliding mode controller and sliding mode observer, Proceedings of IEEE/SICE International Symposium on System Integration (SII2016) (2016b).

Myat Thiri Ko, Yokoyama, M., Nagayoshi, S., Stability analysis of the active pantograph with sliding mode controller and sliding mode observer, Proceedings of $7^{\text {th }}$ International Conference on Science and Engineering (2016c).

Pisano, A. and Usai, E., Contact force regulation in wire-actuated pantographs via variable structure control and frequency-domain techniques, International Journal of Control, 1st series, Vol. 81, No. 11 (2008), pp.1747-1762. 
Pisano, A. and Usai, E., Output-feedback regulation of the contact-force in high-speed train pantographs, Transactions of the ASME, Vol.126, March (2004), pp.82-87.

Sanchez-Rebolloa, C., Jimenez-Octaviob, J. R. and Carnicerob, A., Active control strategy on a catenary-pantograph validated model, Vehicle System Dynamics, Vol. 51, No.4 (2013), pp.554-569.

Shtessel, Y., Edwards, C,. Fridman, L. and Levant, A., Sliding mode control and observation (2014), New York: Birkhauser.

Takeda, T. and Kitamori, T., A design method of linear multi-input-output optimal tracking systems, Transactions of the Society of Instrument and Control Engineers, Vol.14, No.4 (1977), pp.359-364 (in Japanese).

Usuda, T. and Ikeda, M., Estimation method of static height of contact wire using pantograph contact force, RTRI Report, vol. 28, No.10 (2014), pp.35-40 (in Japanese).

Yamashita, Y., Ikeda, M., Masuda, A., Iba, D. and Sone, A., Advanced active control of a contact force between a pantograph and a catenary for a high speed train, Proceedings of $9^{\text {th }}$ World Congress of Railway Research (2011).

Yokoyama, M., Yokoyama, S., Sakakibara, H., Kobayashi, S., Usuda, T. and Ikeda, M., Modeling and robust control of a high speed train pantograph, Bulletin of the JSME, Mechanical Engineering Journal, Vol. 23, No. 3 (2015), paper No.15-00041. 